\title{
Article \\ Genome-Wide Identification and Characterization of AP2/ERF Transcription Factor Family Genes in Oil Palm under Abiotic Stress Conditions
}

\author{
Lixia Zhou ${ }^{*+}$ and Rajesh Yarra ${ }^{+}(\mathbb{C}$ \\ Hainan Key Laboratory of Tropical Oil Crops Biology, Coconut Research Institute, Chinese Academy of Tropical \\ Agricultural Sciences, Wenchang 571339, China; rajeshyarra@rediffmail.com \\ * Correspondence: glzz_2009@163.com \\ + These authors contributed equally.
}

Citation: Zhou, L.; Yarra, R. Genome-Wide Identification and Characterization of $A P 2 / E R F$ Transcription Factor Family Genes in Oil Palm under Abiotic Stress Conditions. Int. J. Mol. Sci. 2021, 22, 2821. https://doi.org/10.3390 /ijms22062821

Academic Editor: Karen Skriver

Received: 6 February 2021

Accepted: 8 March 2021

Published: 10 March 2021

Publisher's Note: MDPI stays neutral with regard to jurisdictional claims in published maps and institutional affiliations.

\begin{abstract}
The $A P 2 / E R F$ transcription factor family members play crucial roles in controlling plant growth and development, as well as responses to various abiotic stresses. Genome-wide identification and characterization of $A P 2 / E R F$ genes has not yet been carried out in the oil palm genome. In the present work, we reported the occurrence of 172 EgAP2/ERFs (AP2, ERF, RAV \& Soloist members) through genome-wide identification. Phylogenetic analysis was used to divide them into four groups, including: $34 \mathrm{AP} 2,131 \mathrm{ERF}, 5 \mathrm{RAV}$, and 2 Soloist gene family members. All 172 AP2/ERF members were unevenly distributed across 16 chromosomes of oil palm. Gene duplication analysis elucidated the tandem duplication of $A P 2 / E R F$ s on chromosome blocks of the oil palm genome during evolution. Gene structure as well as conserved motif analysis demonstrated the conserved nature of intron/exon organization and motifs among the $A P 2 / E R F$ genes. Several cis-regulatory elements-related to hormone, stress, and defense responses-were identified in the promoter regions of $A P 2 / E R F \mathrm{~s}$. Tissue-specific expression of 172 AP2/ERFs in five different tissues of oil palm was also revealed by heatmap analysis using the available transcriptome data. Finally, abiotic stress (salinity, cold \& drought)-responsive $A P 2 / E R F \mathrm{~s}$ in the oil palm genome were validated through $\mathrm{qPCR}$ analysis. Our study provided valuable information on oil palm $A P 2 / E R F$ superfamily members and dissected their role in abiotic stress conditions.
\end{abstract}

Keywords: AP2s; ERFs; stress responses; oil palm; qPCR

\section{Introduction}

Transcription factors are one of the master regulators that control growth, development, and environmental stress responses in plants [1,2]. The APETALA2/ethyleneresponsive element-binding factor $(A P 2 / E R F)$ gene family is one of the largest and most prominent groups of transcription factors present in various plants [3,4]. The AP2/ERF transcription factorss comprise the highly conserved $A P 2 / E R F$ DNA binding domains, with 60-70 amino acid residues. AP2/ERF TFs have been sorted into AP2, ERF (ethyleneresponsive factor), $R A V$ (related to $\mathrm{ABI} / \mathrm{VP} 1$ ), and Soloist families, depending on the number of conserved AP2/ERF domains and sequence similarities [4]. The AP2 family contains either single or double AP2 domains and plays a vital role in developmental processes [5]. The ERF \& dehydration responsive element binding protein (DREB) families contain a single domain of AP2 and are involved in controlling various stress responses [6]. The RAV family consists of AP2 and B3 DNA binding domains, and is known to be involved in biotic and abiotic stress, especially regulated by two hormones (ethylene or brassinosteroids) [7,8]. The Soloist family is the smallest group that contains one AP2 domain and is majorly involved in salicylic acid-mediated defense against pathogens [9].

Based on whole-genome and EST sequence analyses, several researchers identified various numbers of $A P 2 / E R F$ family members in the genomes of a wider range of plants, including: 147 in Arabidopsis [4,10]; 163 in rice [4,11]; 200 in poplar [12]; 132 in grapevine [13]; 
131 in cucumber [14]; 218 in sugarcane [15]; 119 in Chinese jujube [16]; 98 in soybean [17]; 85 in tomato [18]; 117 in wheat [19]; 171 in cauliflower [20]; 134 in California poppy [21]; 193 in orchardgrass [22]; 174 in Ammopiptanthus nanus [23]; and 288 in sunflower [24]. Moreover, reports have described their prominent role in regulating abiotic stress responses (e.g., cold, salinity, drought, heat, and osmotic stress) in various plants [25-30]. Additional reports have demonstrated improved abiotic stress tolerance in transgenic plants via the expression of $A P 2 / E R F$ TFs [31-39]. However, to date, no studies have elucidated the roles of $A P 2 / E R F$ transcription factor members in the Elaeis guineensis genome under abiotic stress. Therefore, it iss important to identify the AP2/ERF TFs in Elaeis guineensis through a genome-wide identification approach.

Oil palm (Elaeis guineensis, Jacq.) is the most prominent oil-yielding tree in the world $[40,41]$. The productivity of oil palm plants is threatened by various environmental stresses, including cold, salinity, drought, and nutritional stress [42-45]. This can lead to substantial economic impact [46]. Therefore, it is necessary to develop oil palm varieties that tolerant to changing environmental conditions via molecular breeding strategies. To date, only a limited number of abiotic stress-responsive genes have been identified in the genome of oil palm. To the best of our knowledge, only WRKY [44] and MYB [42] transcription factors have been identified and partially characterized in response to various abiotic stresses. In view of this, we carried out genome-wide screening in order to identify other important transcription factors (such as AP2/ERF superfamily members) in oil palm. Additionally, expression analyses of identified oil palm AP2/ERF TFs under different abiotic stresses were performed.

In this study, a total of $172 A P 2 / E R F$ transcription family members were identified. In addition, we carried out analyses of the AP2/ERF gene structures, conserved domains, motif composition, phylogenetics, distribution (of $172 \mathrm{TFs}$ on 16 chromosomes), gene duplication, and cis-acting elements. Finally, we investigated the relative expression of 18 EgAP2/ERF genes when oil palm plants were exposed to various abiotic stresses (salinity, drought, and cold) using real-time-PCR analysis. Our results shed light on an important aspect of identification and expression analysis of AP2/ERF transcription factor families under various abiotic stress conditions for the production of abiotic stress-resistant oil palm plants.

\section{Results}

\subsection{Identification of $E g A P 2 / E R F T F s$}

We identified a total of $172 A P 2 / E R F$ genes from the oil palm genome via a bioinformatic approach. The length of EgAP2/ERF genes ranged from 427-3373 bp (Supplementary Table S2). The EgAP2/ERF proteins contained 124-663 of amino acids length (Supplementary Table S2). The identified AP2/ERF genes were categorized into four families (AP2, ERF, $R A V$, and Soloist) depending on the occurrence of conserved AP2 domains. Of the 172 AP2/ERF members identified, 34 of them belonged to the AP2 family, 131 of them belonged to the $E R F$ family, 5 of them belonged to the $R A V$ family, and only 2 belonged to the Soloist family (Supplementary Table S3). The identified number of EgAP2/ERF superfamily gene members (172) was higher than that found in Arabidopsis and rice. Moreover, the number of introns among the four families varied; specifically, the 34 AP2 family members contained more introns (6-10) (Supplementary Table S2) (Figure 1). The ERF family members were mostly without introns, and a few of them contained 1-2 introns (Supplementary Table S2) (Figure 1). RAV family members $E g R A V 01, E g R A V 02$, and $E g R A V 05$ were without introns. EgRAV03 and EgRAV04 had only one intron (Supplementary Table S2) (Figure 1). The remaining two Soloist family members (EgSoloist 1 \& EgSoloist2) had 6 and 7 introns, respectively (Supplementary Table S2) (Figure 1). The length of the individual introns differed in size and showed lengths in the range of 7-19,764 bp long. EgRAV04 had the smallest intron at $7 \mathrm{bp}$. The fourth intron of EgSoloist02 had $19,764 \mathrm{bp}$, which was followed by the intron of EgERF68 with $19,548 \mathrm{bp}$. Our subcellular localization prediction analysis revealed that the majority of $A P 2, E R F, R A V$, and Soloist members localized to extracellular compartments, 
whereas a few members showed dual localization (Supplementary Table S2). However, some of the ERF members were strictly localized to the cytoplasmic region. Interestingly, none of the family members localized to a nuclear region (Supplementary Table S2).

EgAP2.01
EgAP2.02
EgAP2.03
EgAP2.04
EgAP2.05
EgAP2.06
EgAP2.07
EgAP2.08
EgAP2.09
EgAP2.10
EgAP2.11
EgAP2.12
EgAP2.13
EgAP2.14
EgAP2.15
EgAP2.16
EgAP2.17
EgAP2.18
EgAP2.19
EgAP2.20
EgAP2.21
EgAP2.22
EgAP2.23
EgAP2.24
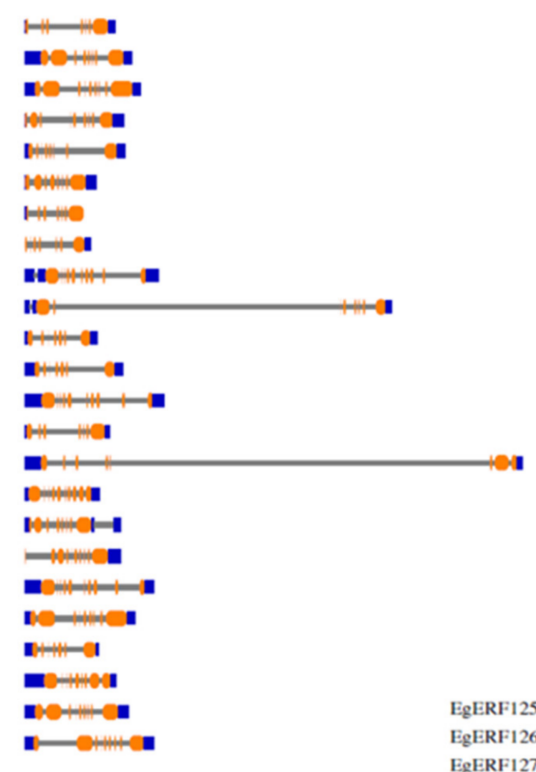

EqERF103

EgERF104

EgERF105

EqERF106

EqERF107

EgERF108

EgERF109

EgERF11
EgERF110

EgERF110

EqERF112

EgERF113

EgERF114

EqERFIIS

EgERF116

EgAP2.14

EgAP2.16

EqERF117

EgAP2.17
EgAP2.18

EgAP2.19

EgAP2.20

EgAP2.22

EgAP2 24
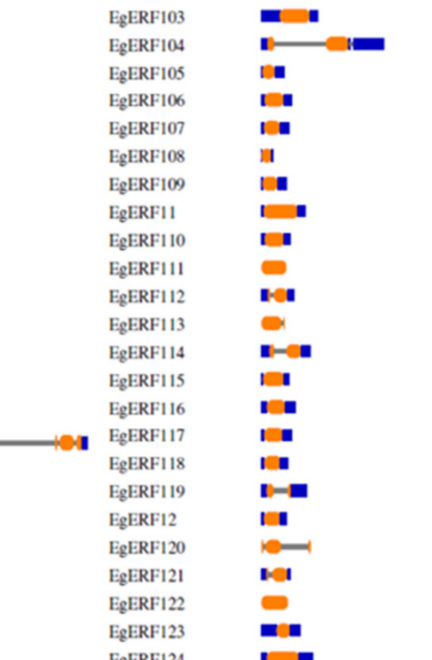

EgERF30

EgERF33

目

1

1

i-a

110

10

1

:-

in-

10.

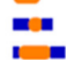

EgERF125

EgERF126

EgERF127

EgERF129

EgERF13

EgERF130

EgERF131

EgERF14

EgERF15

EgERF16

EgERF17

EgERF19

EgERF20

EgERF21

EgERF22

EgERF23

EgERF24

EgERF25

EgERF26

EgERF27

EgERF28

EgERF29

EgERF34

EgERF35

EgERF 36

EgERF37

EgERF38

EgERF39

EgERF40

EgERF4I

EgERF42

EgERF43

EgERF44

EgERF45

EgERF46

EgERF47

EgERF48

EgERF49

EgERF50

EgerEF5!

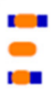

I. EgERFS4

-D EgERF55

I. EgERF56

DE EgERPS8

e EqERF59

El EqERF60

EgERF62

MI EgERF63

E. Egerfo4

agerfos

-1. EgerFo6

EgERF67
EgERF68

EgERFG

EgERFT0

EgERFI

EgerF73

IO EgERF/4

EgERF75

EgERF76

EgERF52

EgERF53

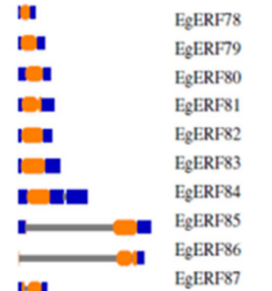

EgerF88

EgERF89

EgERP90

II EgERP91

D EgERP92

1. EgERP94

-1 EgERP95

EgERP96

IN EgERP97

n. EgERP98

H. EgERP99

11

11

1

5

1

a.

m.

MI

av

-1-

E.

1

10.

$-$

a1

19.

11เ-

in-1

ra

10

10

1

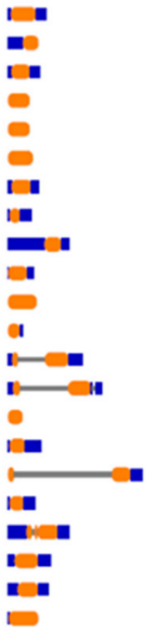

$\begin{array}{ll}\text { EgRAV01 } & \text { EgRAV01 } \\ \text { EgRAV02 } & \text { EgRAV02 } \\ \text { EgRAV03 } & \text { EgRAV03 } \\ \text { EgRAV04 } & \text { EgRAV04 } \\ \text { EgRAV05 } & \text { EgRAV05 } \\ \text { EgSoloist01 } & \text { EgSoloist01 } \\ \text { EgSolois02 } & \text { EgSolois02 }\end{array}$

$=1$

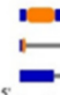

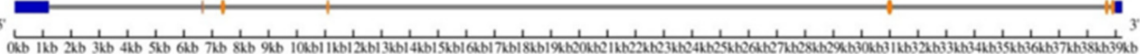

CDS

Intron

5'UTR

3'UTR

Figure 1. Intron-exon organization of 172 AP2/ERF transcription factors in the oil palm genome. Coding sequences (CDS) are represented by orange color blocks; $3^{\prime} \& 5^{\prime}$ un-translated (UTR)regions are represented by grey color blocks; intron regions are represented by grey color blocks.

\subsection{Phylogenetic and Conserved Motif Analysis of EgAP2/ERF TFs}

A phylogenetic tree was constructed using the deduced protein sequences of AP2/ERF genes from oil palm, rice, and Arabidopsis. The obtained phylogenetic tree grouped all 
of them into four main categories of $A P 2 / E R F$ gene families (AP2, ERF, $R A V$, and Soloist) (Figure 2). Most of the sequences from oil palm, rice, and Arabidopsis grouped under $E R F$ (followed by AP2) (Figure 2). A similar number of genes from oil palm, rice, and Arabidopsis clustered into the $R A V$ family group. Moreover, the Soloist family had more numbers from oil palm (2) than Arabidopsis (1) and rice (1) (Figure 2). Furthermore, we analyzed the motif composition in each AP2/ERF family member using the online Multiple Expectation maximizations for Motif Elicitation (MEME) tool. Motif analysis revealed the occurrence of ten conserved motifs (1-10) from the identified oil palm AP2/ERF TFs (Figure 3). Results showed the existence of motif 4 and motif 5 in all four families of EgAP2/ERF protein sequences (Figure 3). Motif 1, 2, 3, and 7 existed only in AP2 family members, whereas motif 6 was present in all except Soloist family members. Motif 10 was present only in $R A V$ family members (Figure 3). The Soloist family members only contained two motifs (4 and 5). Motif 9 was restricted to ERF family members. The amino acid composition of each motif is illustrated in Figure 3.

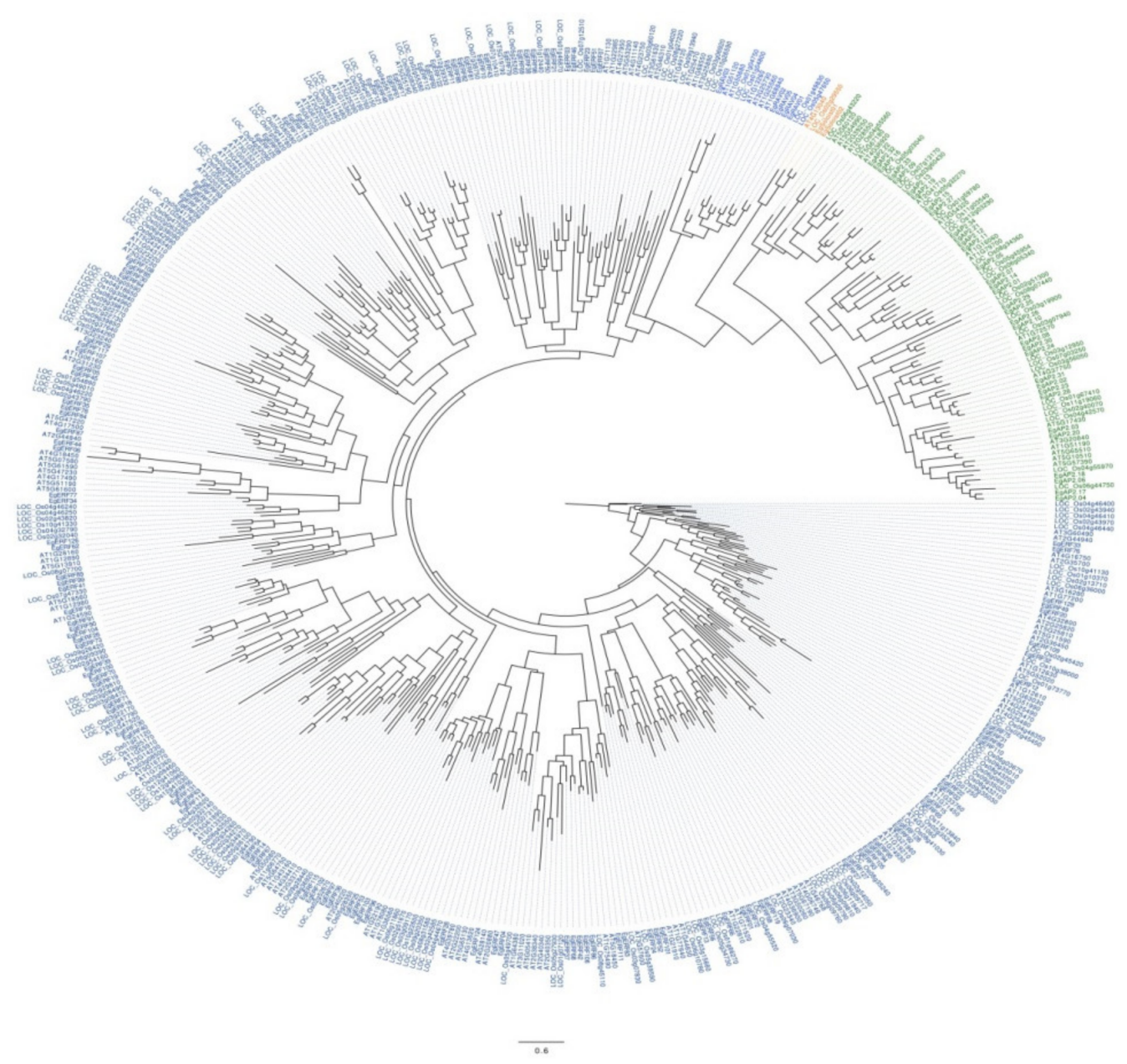

Figure 2. Phylogenetic analysis of $A P 2 / E R F(\mathrm{AP2}, \mathrm{ERF}, R A V$, and Soloist) family members in oil palm, Arabidopsis, and Oryza sativa. Each of the families from oil palm, Arabidopsis, and rice are grouped and represented in various colors (Navy blue, blue, orange, and green). 


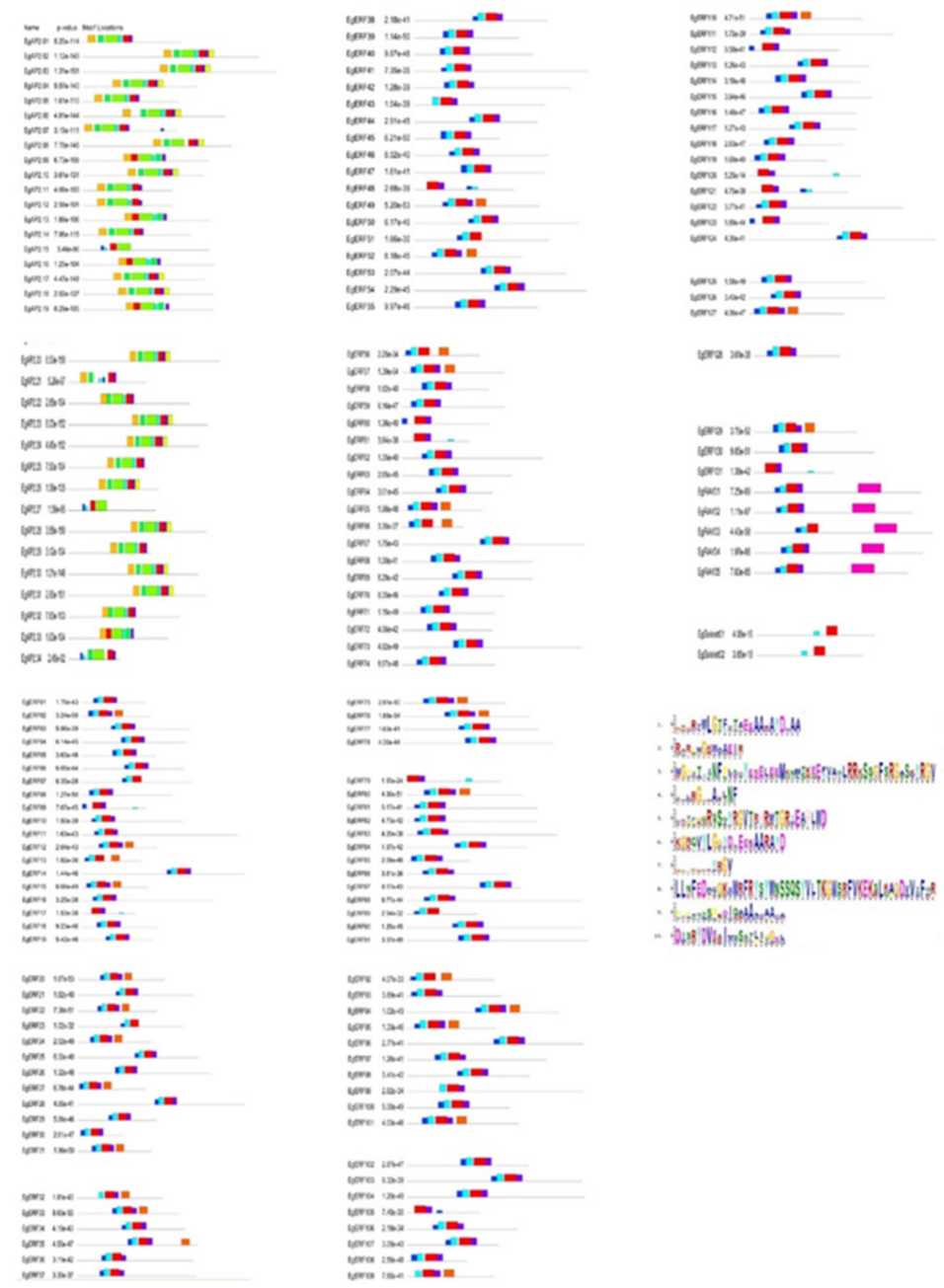

Figure 3. The architecture of conserved motifs in oil palm AP2/ERF proteins. Ten motifs were identified in AP2/ERF proteins using the MEME tool. Each motif in AP2/ERF proteins is represented with different colors. The abundance of each amino acid in every motif of oil palm AP2/ERF proteins is given in the sequence logo.

\subsection{Oil Palm AP2/ERF Genes Duplication Analysis}

The gene duplication events analysis of AP2/ERF family members in the oil palm genome was examined via MCScanx and Circos software tools. The substitution ratios $(\mathrm{ka} / \mathrm{ks})(\mathrm{ka} / \mathrm{ks}>1$, positive selection; $\mathrm{ka} / \mathrm{ks}=1$, neutral selection; $\mathrm{ka} / \mathrm{ks}<1$, negative selection) were calculated in order to analyze the AP2/ERF gene duplication. The calculated $\mathrm{Ka} / \mathrm{Ks}$ ratio values of all the $A P 2 / E R F$ genes were found to be less than one $(<1)$ (Supplementary Table S4). These results indicated that the evolution of oil palm AP2/ERF genes happened via strong purifying selection. Moreover, EgAP2/ERF family members experienced tandem duplications across sixteen chromosomes during evolution (Figure 4). Furthermore, all 16 chromosomes of oil palm genome contained duplicated AP2/ERF family members (Figure 4). Chromosomes 3, 5, 13, 14, and 15 had more duplicated AP2/ERF gene pairs, whereas chromosomes 9 and 11 contained less. (Figure 4). These results demonstrate the prominent role of tandem duplication in the expansion of AP2/ERF gene family members in the oil palm genome. 


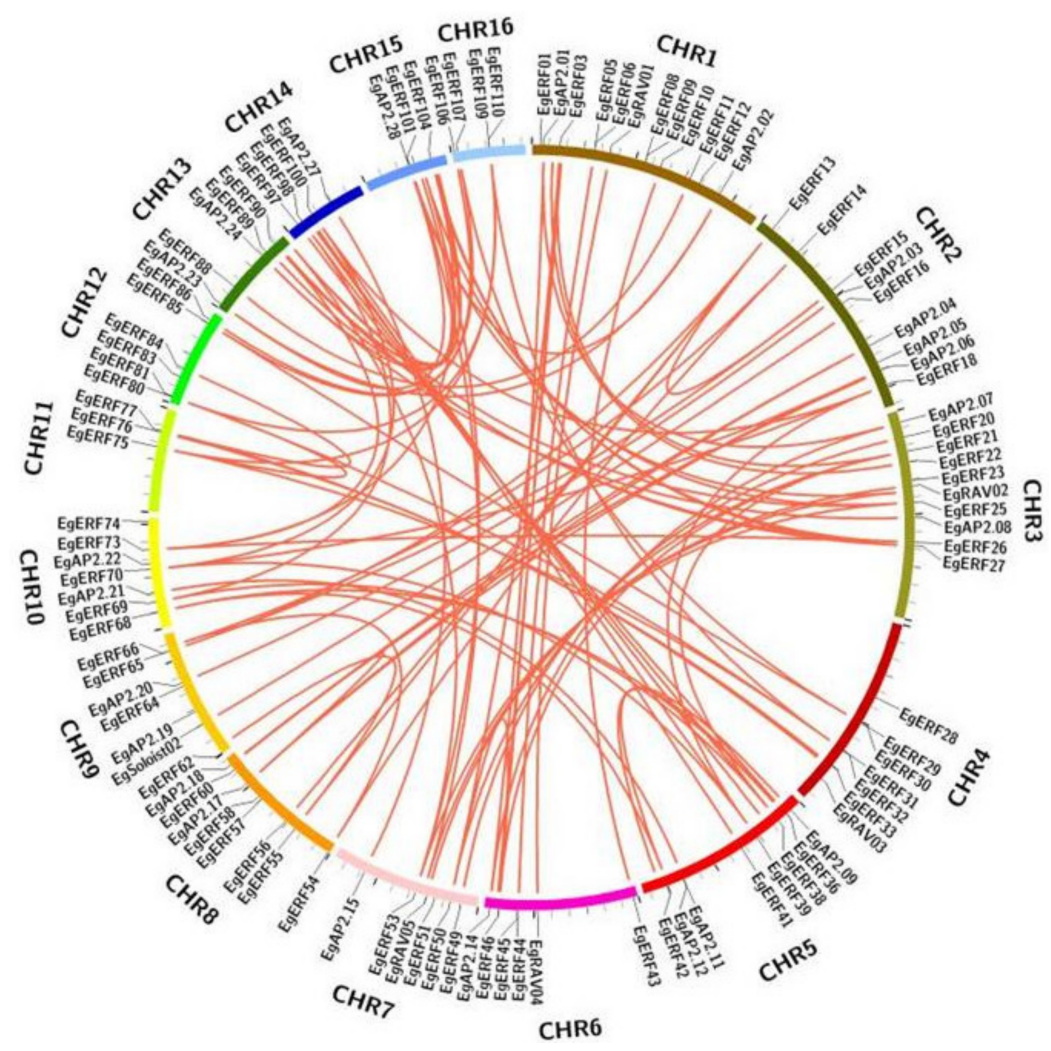

Figure 4. Schematic interpretation of AP2, ERF, $R A V$, and Soloist genes' duplication in the oil palm genome. Each chromosome block is denoted with a different color. Red lines inside the schematic view denote the duplicated gene pairs of oil palm AP2/ERFs.

\subsection{Chromosomal Distribution of 172 EgAP2/ERF TFs}

All 172 identified EgAP2/ERF genes were mapped to 16 chromosomes of oil palm. The distribution of $\mathrm{Eg} A P 2 / E R F s$ varied through 16 chromosomes of oil palm. Most (15) EgAP2/ERFs were mapped on chromosome 1, followed by chromosome 2 (12) and chromosome 5 (12) (Figure 5). Chromosome 13 had the lowest number (2) of EgAP2/ERFs (Figure 5). None of the AP2 family members were mapped to chromosomes 4, 11, 12, or 16; however, ERF family members were distributed on all 16 chromosomes of oil palm (Figure 5). Moreover, $R A V$ family members were only mapped to chromosomes 3, 4, 6, 7 and 11-whereas Soloist family members were located only on chromosomes 2 and 9.

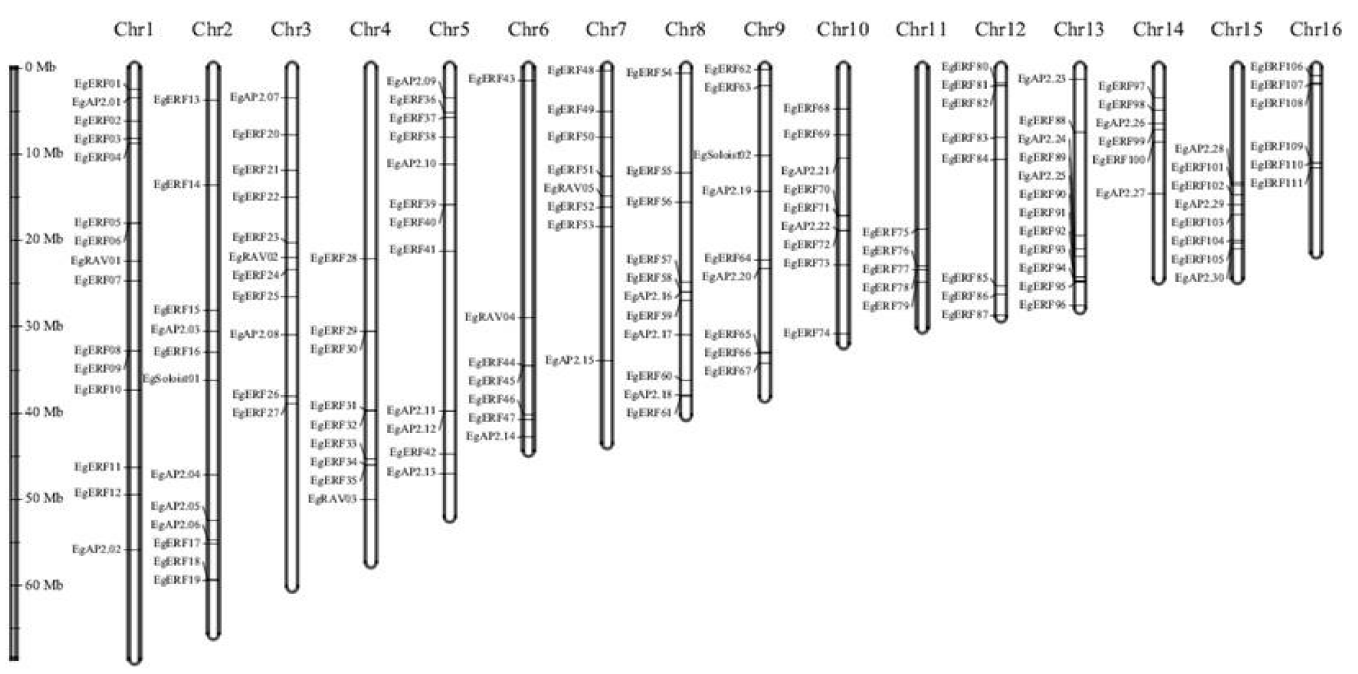

Figure 5. Chromosomal mapping of 172 oil palm AP2/ERFs. The length of the oil palm chromosomes is indicated on the vertical greyscale. The chromosome numbers (1-16) are indicated on the top of each chromosome. 


\subsection{Analysis of Cis-Acting Elements in Promoter Regions of EgAP2/ERFs}

We analyzed the presence of various cis-acting elements located in the promoter regions of EgAP2/ERFs using the PlantCare database. We found various cis-regulatory elements, related to the gibberellin response, defense response, stress response, and low temperature response. Additionally, those elements were involved in the regulation of cell cycle and seed-specific expression (Figure 6). However, the majority of EgAP2/ERFs possessed cis-regulatory elements (CREs) responsible for the regulation of cell cycle and stress/defense responses (Figure 6). Most of the AP2 and ERF family members contained hormone, stress, and defense-responsive regulating elements. The Soloist family members contained stress-responsive elements, mostly confined to low-temperature stressresponsive elements (Figure 6). The RAV family members contained cell cycle regulating and stress-responsive elements but were mostly confined to CREs of cell cycle regulation (Figure 6).
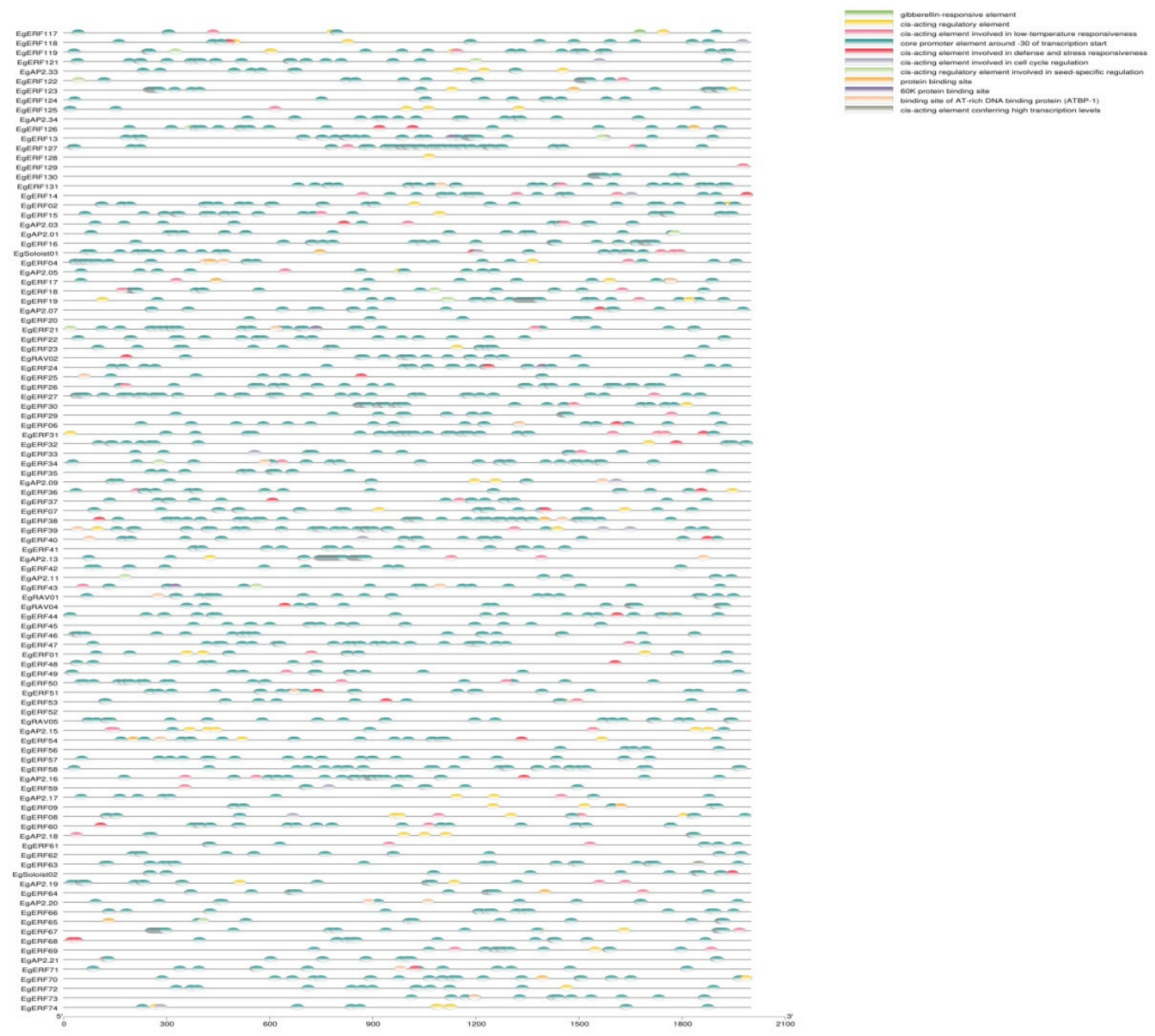

Figure 6. Schematic representations of cis-regulatory elements present in $A P 2 / E R F$ s as investigated via the PlantCARE tool.

\subsection{Transcriptome Data-Based Tissue-Specific Expression Profiling of EgAP2/ERFs}

We analyzed the expression profiles of $172 \mathrm{Eg} A P 2 / E R F \mathrm{~s}$ in six different tissues (leaf, root, fruit, flower, shoot, and mesocarp $(15,17,21$ and 23 weeks old $)$ ) of oil palm plants (unstressed) from the available transcriptome data (SRR851096, SRR851071, SRR851067, SRR851108, SRR851103, SRR190698, SRR190699, SRR190701 \& SRR190702). Heatmap analysis showed that the majority of the genes were expressed at a lower level in oil palm tissues (Figure 7). A total of seven genes (EgERF27, EgERF31, EgERF34, EgERF75, EgERF80, $E g E R F 102$ \& EgERF115) with the highest expressions were observed in both leaf and root tissues of oil palm (Figure 7). EgERF26, EgERF61, and EgERF73 showed higher expression in mesocarp tissues of 21 week-old oil palm (Figure 7). However, the majority of the gene 
expression was confined to the root, leaf, and fruit of oil palm. A higher number of genes showed a moderate level of expression in flowers. The majority of the AP2 members were down-regulated in oil palm tissues. The RAV members (such as EgRAV01 and EgRAV02) displayed the highest levels of expression in flower and fruit tissues, respectively (Figure 7). The EgSoloist02 gene expressed in leaf tissue of oil palm. Overall, it was indicated that ERF, AP2, RAV, and Soloist family members exhibited tissue-specific expression in oil palm (Figure 7).

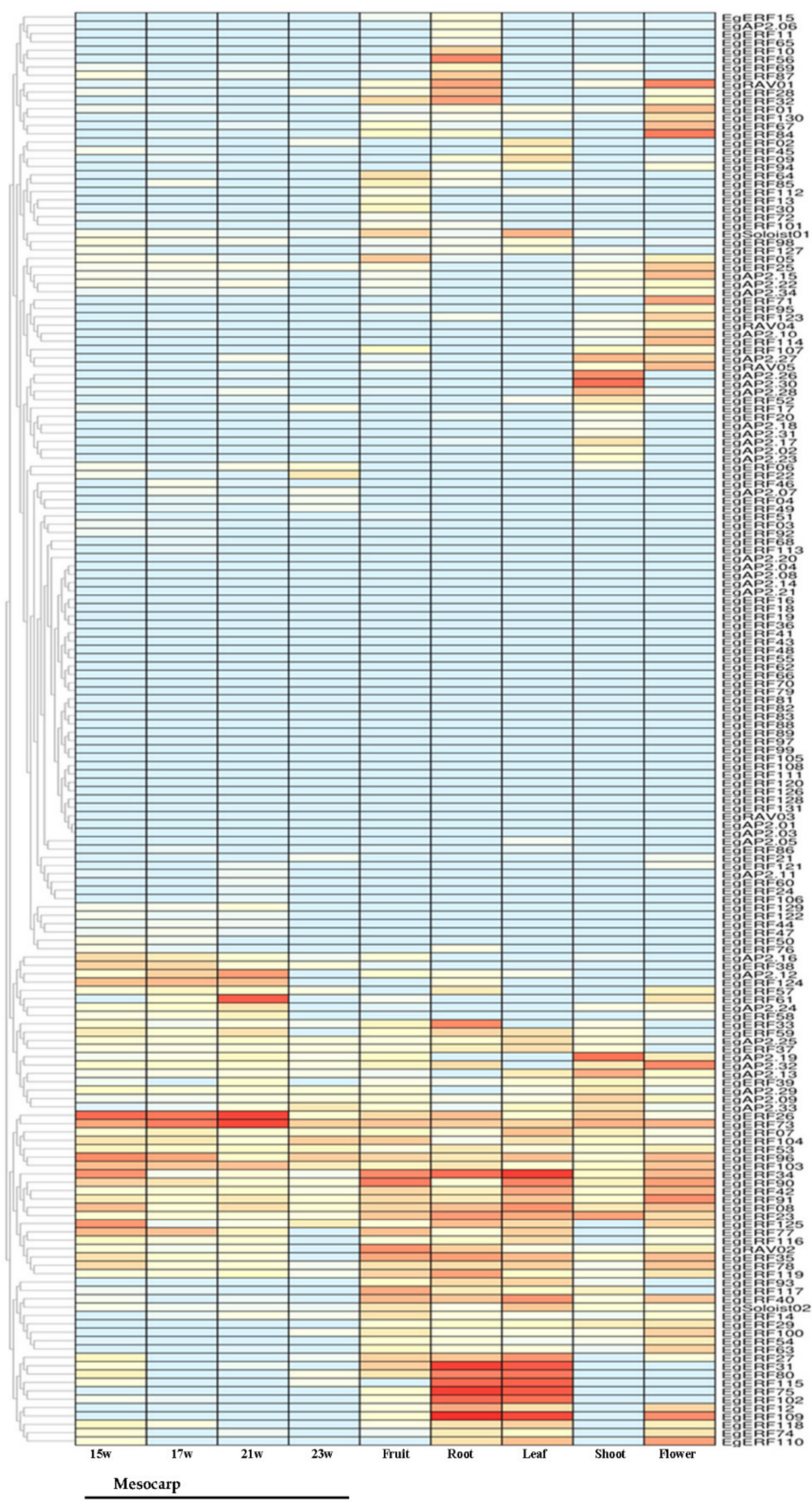

Figure 7. Heatmap for $172 \mathrm{Eg} A P 2 / E R F$ gene expression in mesocarp (15, 17, 21 \& 23 weeks old), root, shoot, leaf, flower, and fruit tissues of oil palm. Transcriptome data representation numbers (SRR190698, mesocarp (15 weeks); SRR190699, mesocarp (17 weeks); SRR190701, mesocarp (21 weeks); SRR190702, mesocarp (23 weeks); SRR851108, flower; SRR851067, fruit; SRR851096, leaf; SRR851071, root; SRR851103, shoot) are labeled in the bottom of the heatmap. The color scale represents the expression levels (low or high) of $A P 2 / E R F$ s. 


\subsection{EgAP2/ERF Genes' Expression Analysis under Abiotic Stress}

To further explore the responses of EgAP2/ERF genes under abiotic stress conditions (salinity, drought, and cold), we examined the expression patterns of $18 \mathrm{Eg} A P 2 / E R F$ genes (EgAP2.25, EgAP232, EgAP2 34, EgERF07, EgERF14, EgERF23, EgERF26, EgERF42, EgERF73, $E g E R F 90, E g E R F 104, E g E R F 125, E g E R F 129, E g E R F 130$, and $E g R A V 02)$ from three different families via RT-qPCR analysis (Figure 8). Interestingly, all of the tested oil palm AP2/ERF gene family members were placed under salinity, drought, and cold stress conditions at a certain time ( $4 \mathrm{~h}, 24 \mathrm{~h} \& 48 \mathrm{~h})$. All of the salinity stress treatments $(4 \mathrm{~h}, 24 \mathrm{~h}, 48 \mathrm{~h}$ ) induced the expression of $E g E R F 14, E g E R F 73$, and $E g R A V 02$ genes (Figure 8). The drought stress exposure at all time intervals $(4 \mathrm{~h}, 24 \mathrm{~h}$, and $48 \mathrm{~h})$ induced the expression of $E g A P 2.09$, EgERF26, EgERF90, and EgERF104 genes (Figure 8). The EgAP2.15, EgAP2.34, EgERF23, $E g E R F 104$, and $E g E R F 130$ genes showed increased expression under cold stress exposure ( $4 \mathrm{~h}, 24 \mathrm{~h}$, and $48 \mathrm{~h}$ ) (Figure 8). Our abiotic stress-responsive expression analysis results indicated the potential role of $A P 2 / E R F$ family genes in regulating abiotic stress responses in oil palm.

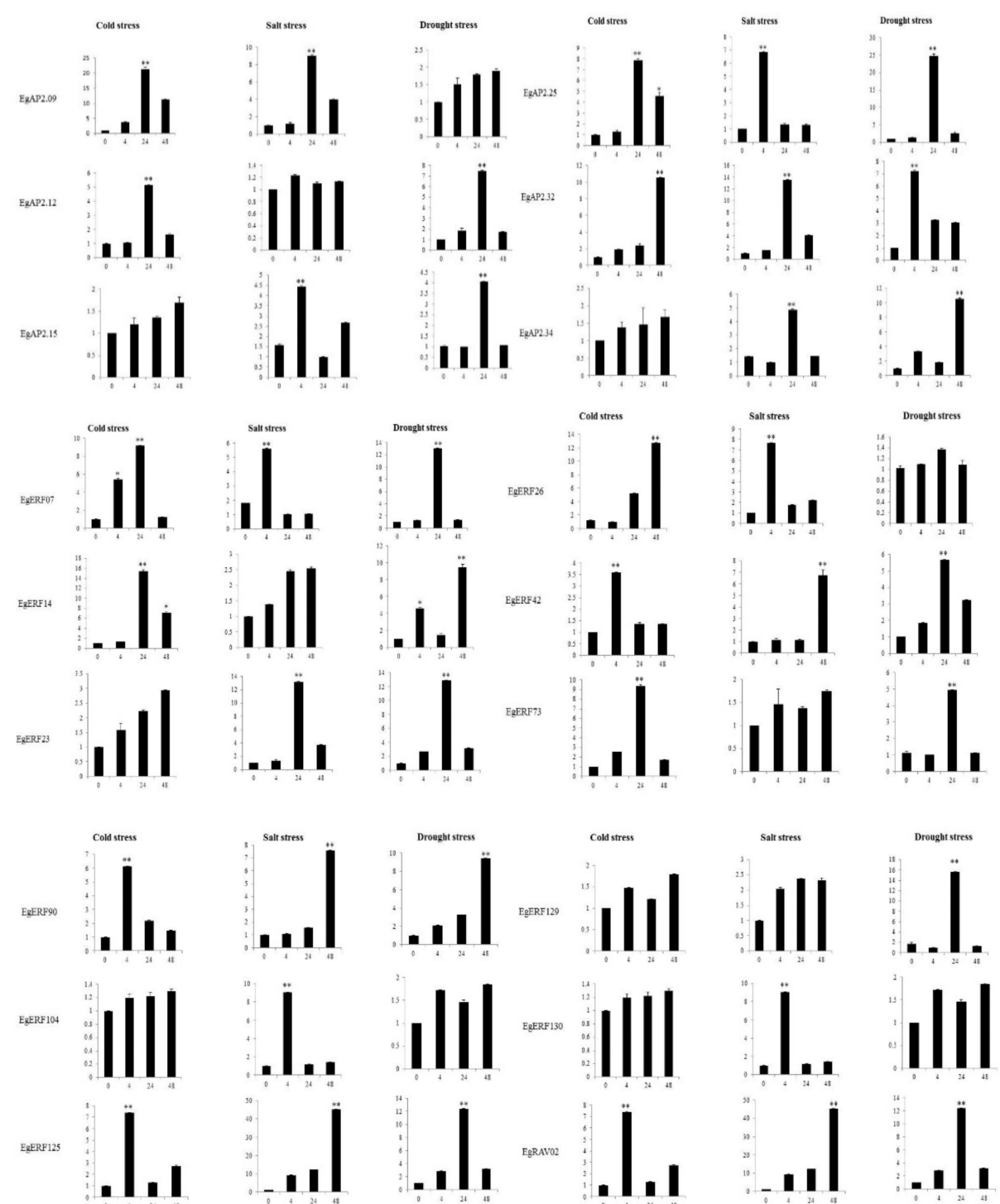

Figure 8. Real-time PCR analysis of $18 A P 2 / E R F$ transcription factors in response to cold, salt, and drought stress $(0,4,24 \& 48 \mathrm{~h})$ conditions. $X$-axis represents the hours of stress exposure. $Y$-axis represents the relative expression of a transcription factor. Data represent the mean $\pm \mathrm{SE}$ of three replicates. Asterisks represent significant difference at $p \leq 0.05\left(^{*}\right)$ and $p \leq 0.01\left(^{* *}\right)$. 


\section{Discussion}

$A P 2 / E R F$ superfamily genes act as vital regulators for governing various physiological and stress responses in a wide range of plants [25-30]. Few genome-wide studies have been carried out to identify AP2/ERF family members in plant genomes [4,10,11,13-24]. To date, no genome-wide studies have been conducted to explore the structure and role of $A P 2 / E R F$ family genes in oil palm. Ours is thus the first report on the identification of $A P 2 / E R F$ transcription factors in oil palm. In addition, we explored their role in various abiotic stress responses based on real-time PCR analysis.

The identified number (172) of AP2/ERFs in the E. guineensis genome was greater than the identified number of $A P 2 / E R F$ members in various plants, but less than the number of AP2/ERF genes in poplar (200) [12], sugarcane (218) [15], orchardgrass(193) [23], Ammopiptanthus nanus (174) [22], and sunflower (288) [24] genomes. The oil palm AP2/ERF gene structural analysis revealed that a higher number of AP2 family members contained more introns (6-10) compared to ERF, RAV or Soloist family members. However, the occurrence of introns among all family members was very low. Our oil palm AP2/ERF gene structure analysis results were also consistent with other AP2/ERFs structural organizations observed in various plants [21,47-49]. Conserved motifs analysis was used to determine transcriptional activity, protein-protein interactions, and DNA-binding activity of TFs [50]. The conserved motif analysis of all $172 \mathrm{Eg} A P 2 / E R F$ family members revealed the occurrence of 10 conserved motifs; those motifs might be related to specific functions shared among EgAP2/ERF family members. Phylogenetic analysis of 172 oil palm AP2/ERF family members alongside Arabidopsis and rice indicated the conservation and diversification of $\mathrm{AP} 2$, ERF, $R A V$, and Soloist members among model-to-crop plants. Gene duplication events in plants commonly occur via tandem, whole-genome or segmental duplications [51]. Our study demonstrated the occurrence of tandem duplications in oil palm for the expansion of all $A P 2 / E R F$ family members during evolution. These results also agreed with previous findings of the tandem duplications of AP2/ERF members in Saccharum spontaneum L. [15].

Several studies demonstrated that AP2/ERF family members were widely expressed in various tissues/organs for regulating the growth and development of plants $[15,16,51]$. The tissue-specific expression profiling data of genes is vital for elucidating the functional roles of genes. Our heatmaps analysis provided information on the expression of AP2, ERF, $R A V$, and Soloist members in the root, shoot, leaf, flower, and fruit mesocarp of oil palm plants. Each of the AP2/ERF family members (AP2, ERF, RAV, and Soloist) showed tissue-specific expression. However, the majority of them were expressed in all tissues, indicating their role in governing growth and developmental processes in oil palm plants.

Previous studies demonstrated the regulatory role of AP2/ERF members in response to various abiotic stress conditions $[15,28-30,51,52]$. Here, we examined the relative expression of $18 \mathrm{Eg} A P 2 / E R F$ family members, including three AP2 genes (EgAP2.25, EgAP232, EgAP2 34), eleven ERF genes (EgERF07, EgERF14, EgERF23, EgERF26, EgERF42, EgERF73, $E g E R F 90, E g E R F 104, E g E R F 125, E g E R F 129, E g E R F 130)$ and one $R A V$ family gene (EgRAV02) under salinity, cold, and drought stress conditions. All the tested transcription factors showed expression under all three abiotic stresses. The present study showed a higher expression of $E g E R F 14, E g E R F 73$, and $E g R A V 02$ genes under salinity stress environments. Our results corroborated earlier reports on salt stress-induced ERF and $R A V$ family members in various plants [15,52-54]. Higher expressions of EgAP2.09, EgERF26, EgERF90, and $E g E R F 104$ genes were also observed under drought stress conditions. The drought stress-induced AP2 and ERF family members were also reported in earlier studies and our results supported their findings $[20,28,51,53]$. In our study, cold stress induced the highest expressions of $E g A P 2.15, E g A P 2.34, E g E R F 23, E g E R F 104$, and $E g E R F 130$ genes; these results are in agreement with previous reports on cold-induced $A P 2$ and $E R F$ transcription factors $[24,28,53,55]$. These findings will be helpful in choosing potential AP2/ERF family members for the production of abiotic stress-tolerant oil palm varieties. 


\section{Materials and Methods}

\subsection{Database Search and Identification of AP2/ERF TFs in Oil Palm Genome}

The genome sequences of African oil palm (Elaeis guineensis, Jacq.) were retrieved from the National Center for Biotechnology Information (NCBI) database. The known AP2/ERF sequences of Arabidopsis thaliana and Oryza sativa (ssp. Japonia and Indica) were downloaded from the Plntfdb (http://plntfdb.bio.uni-potsdam.de/v3.0/, accessed on 28 January 2021) and PlantTFDB (http:/ / planttfdb.gao-lab.org/, accessed on 28 January 2021) databases and used as query sequences in the Basic Local Alignment Search Tool (BLAST) program to search for AP2/ERF genes in the oil palm genome. The conserved AP2 domains (PF00847) corresponding to AP2/ERF gene family members were obtained from the PFAM database (https:/ / pfam.xfam.org/, accessed on 28 January 2021). The conserved domains database (CDD) online tool (https: / /www.ncbi.nlm.nih.gov/cdd/, accessed on 28 January 2021) was employed to verify the candidate gene sequences obtained from PFAM. A total of $172 \mathrm{Eg} A P 2 / E R F$ genes were finally identified from the genome. The ExPASy proteomic website (https:/ / web.expasy.org/compute_pi/, accessed on 28 January 2021) was employed to predict the molecular weight and isoelectric points of oil palm $A P 2 / E R F$ proteins. Subcellular localization of all identified $172 \mathrm{Eg} A P 2 / E R F$ proteins was predicted with the help of the CELLO tool (http:/ / cello.life.nctu.edu.tw/, accessed on 28 January 2021).

\subsection{EgAP2/ERF Gene Structure and Conserved Motif Analysis}

Intron-exon organization of EgAP2/ERFs was investigated by using the GSDS-2.0 online program (http:/ / gsds.gao-lab.org/, accessed on 28 January 2021). The conserved motifs that existed in the identified AP2/ERF proteins were explored with the help of the Motif Elicitation (MEME) online program (http:/ / meme-suite.org/tools/meme, accessed on 28 January 2021).

\subsection{Phylogenetic Analysis, Chromosomal Distribution and Duplication Events Analysis of Oil Palm AP2/ERF Genes}

The evolutionary relationships among AP2/ERF protein sequences of Elaeis guineensis (172 AP2/ERFs), Arabidopsis thaliana, and Oryza sativa (ssp. Japonia and Indica) were established by constructing a phylogenetic tree via the neighbor-joining method using MEGA 6.06 software. Various parameters (e.g., poison correction, pairwise deletion, and 1000 bootstrap replicates) were used to construct the phylogenetic tree. The chromosomal distribution of $172 \mathrm{Eg} A P 2 / E R F$ s was investigated against the oil palm genome using th e TBtools software (https://github.com/CJ-Chen/TBtools, accessed on 28 January 2021). AP2/ERF gene duplication events were analyzed using the MCScanX tool (Multiple Collinearity Scan) with a set of parameters [56].

\section{4. cis-Acting Elements Analysis of EgAP2/ERFs}

The potential cis-regulatory elements of oil palm $A P 2 / E R F$ s were checked by choosing $200 \mathrm{bp}$ upstream of transcription start site TSS in each of AP2/ERF gene. We employed the PlantCARE online tool (http:/ / bioinformatics.psb.ugent.be/webtools/plantcare/html/, accessed on 28 January 2021) to identify the cis-acting regulatory elements.

\subsection{Source of Transcriptome Data and Tissue-Specific Expression Analysis of EgAP2/ERF TFs}

The transcriptome data of oil palm tissues-including leaf, root, fruit, flower, shoot, and mesocarp $(15,17,21$, and 23 weeks old) - were downloaded from the SRA (Sequence Read Archive) database of the NCBI website. The transcript abundance of EgAP2/ERFs in different tissues was calculated with RPKM (reads per kilobase per million mapped reads) values. The heatmap tool was used to generate a heatmap of all $172 \mathrm{Eg} A P 2 / E R F \mathrm{~s}$. 


\subsection{Oil Palm Plant Materials and Stress Exposure}

The African oil palm (Elaeis guineensis Jacq.) plantlets were raised in greenhouses $\left(27^{\circ} \mathrm{C} /\right.$ temperature; $16 \mathrm{~h} /$ light; $8 \mathrm{~h} /$ darkness; humidity of about $\left.50-60 \%\right)$ at Coconut Research Institute, CATAS, Wenchang, China. Healthy oil palm plantlets of the same age (6 months old) were chosen to undergo abiotic stress treatments (cold, salt, and drought). Before stress exposure, all plants were shifted to a growth chamber at $27^{\circ} \mathrm{C}$ for one day. Each abiotic stress treatment (cold or drought or salinity) was done after $0 \mathrm{~h}$ (control), $4 \mathrm{~h}$, $24 \mathrm{~h}$, and $48 \mathrm{~h}$, using different seedlings ( 9 for each treatment). Oil palm seedlings were exposed to cold stress exposure at $8{ }^{\circ} \mathrm{C}$. Drought stress condition for the oil palm seedlings was established after reaching $20 \%$ water content in the soil. Salinity stress was induced by immersing the roots of oil palm seedlings in $300 \mathrm{mmol} / \mathrm{L}$ concentration of $\mathrm{NaCl}$ solution. At different time intervals $(0,4,24 \& 48 \mathrm{~h})$ of the above stress exposures, the spear leaves were collected and immediately frozen in liquid nitrogen for further RT-qPCR analysis. In addition, oil palm seedlings for control experiments were maintained under light $(16 \mathrm{~h})$ and darkness $(8 \mathrm{~h})$ at $27^{\circ} \mathrm{C}$. All the stress experiments used three biological replicates and were repeated three times.

\subsection{Quantitative Real-Time PCR (RT-qPCR) Analysis of EgAP2/ERFs}

Total RNA was extracted from leaf samples collected before and after stress treatments, following the previously reported protocol (Iqbal et al., 2009). The isolated RNA $(\sim 2 \mu \mathrm{g})$ was then reverse transcribed to cDNA using a MightyScript Plus first-strand cDNA synthesis kit for analyzing the relative expression of EgAP2/ERFs under abiotic stress conditions. The RTqPCR amplifications were executed in a Mastercycler ${ }^{\circledR}$ ep realplex Real-time PCR System in 384-well optical plates. A high-throughput qPCR primer designing tool (Quantprime (https: / / quantprime.mpimp-golm.mpg.de/, accessed on 28 January 2021)) was used to design AP2/ERF gene-specific primers. The thermal conditions for amplification reactions were set at $95^{\circ} \mathrm{C} / 5 \mathrm{~s}, 58^{\circ} \mathrm{C} / 15 \mathrm{~s}$, and $68^{\circ} \mathrm{C} / 20 \mathrm{~s}$. All the amplifications were done with three biological and three technical repeats. The $2^{-\Delta \Delta \mathrm{Ct}}$ method was used to calculate the relative expression of all tested $A P 2 / E R F$ genes. The list of qPCR primers used in this study is provided in Supplementary Table S1.

\subsection{Statistical Analysis}

The experiments were performed with three technical and biological replications. One-way analysis of variance (ANOVA) was used to determine the statistical significance at the $p \leq 0.05$ and $p \leq 0.01$ levels. Asterisks represent a significant difference at $p \leq 0.05\left(^{*}\right)$ and $p \leq 0.01(* *)$.

\section{Conclusions}

A total of $172 A P 2 / E R F$ family TFs were identified in the African oil palm genome through bioinformatic analysis. Our bioinformatic approach revealed the AP2/ERF genes' structural organization, distribution across 16 chromosomes, motif conservation among all the family members, and duplication events of all AP2, ERF, $R A V$, and Soloist gene family members. Furthermore, we predicted subcellular localization and analyzed the cis-acting regulatory elements in promoter regions and tissue-specific expression of oil palm AP2/ERF family members. Additionally, our experimental approach validated the role AP2/ERF TFs play in various abiotic stress conditions. Overall, our bioinformatic and experimental approaches have provided valuable information on oil palm AP2/ERF genes-information that can be used for the development of abiotic stress-tolerant oil palm varieties in the near future.

Supplementary Materials: The following are available online at https:/ / www.mdpi.com/1422-006 7/22/6/2821/s1, Table S1; Table S2; Table S3; Table S4. 
Author Contributions: Concept, L.Z.; methodology and data analysis, L.Z. and R.Y.; writingoriginal draft preparation, R.Y.; writing - review and editing, R.Y.; supervision, L.Z. All authors have read and agreed to the published version of the manuscript.

Funding: This research work was financially supported by the National Natural Science Foundation of China (No: 31870670) with basic support from the Chinese Academy of Tropical Agricultural Sciences, China.

Institutional Review Board Statement: Not applicable.

Informed Consent Statement: Not applicable.

Data Availability Statement: The data presented in this study are available in supplementary file.

Conflicts of Interest: The authors declare no conflict of interest.

\section{References}

1. Baillo, E.H.; Kimotho, R.N.; Zhang, Z.; Xu, P. Transcription Factors Associated with Abiotic and Biotic Stress Tolerance and Their Potential for Crops Improvement. Genes 2019, 10, 771. [CrossRef] [PubMed]

2. Khan, S.A.; Li, M.Z.; Wang, S.M.; Yin, H.J. Revisiting the Role of Plant Transcription Factors in the Battle against Abiotic Stress. Int. J. Mol. Sci. 2018, 19, 1634. [CrossRef] [PubMed]

3. Feng, K.; Hou, X.L.; Xing, G.M.; Liu, J.X.; Duan, A.Q.; Xu, Z.S.; Li, M.Y.; Zhuang, J.; Xiong, A.S. Advances in AP2 / ERF super-family transcription factors in plant. Crit. Rev. Biotech. 2020, 40, 750-776. [CrossRef]

4. Nakano, T.; Suzuki, K.; Fujimura, T.; Shinshi, H. Genome-wideanalysis of the ERF gene family in Arabidopsis and rice. Plant Physiol. 2006, 140, 411-432. [CrossRef]

5. El Ouakfaoui, S.; Schnel, 1.J.; Abdeen, A.; Colville, A.; Labbe, H.; Han, S.; Baum, B.; Laberge, S.; Miki, B. Control of somatic embryogenesis and embryo development by AP2 transcription factors. Plant Mol. Biol. 2010, 74, 313-326. [CrossRef]

6. Mizoi, J.; Shinozaki, K.; Yamaguchi-Shinozaki, K. AP2/ERF family transcription factors in plant abiotic stress responses. Biochim. Biophys. Acta 2012, 1819, 86-96. [CrossRef] [PubMed]

7. Je, B.I.; Piao, H.L.; Park, S.J.; Park, S.H.; Kim, C.M.; Xuan, Y.H.; Park, S.H.; Huang, J.; Do Choi, Y.; An, G. RAV-Like1 maintains brassinosteroid homeostasis via the coordinated activation of BRI1 and biosynthetic genes in rice. Plant Cell 2010, 2, 1777-1791. [CrossRef]

8. Li, C.W.; Su, R.C.; Cheng, C.P.; Sanjaya, Y.S.J.; Hsieh, T.H.; Chao, T.C.; Chan, M.T. Tomato RAV transcription factor is a pivotal modulator involved in the AP2/EREBP-mediated defense pathway. Plant Physiol. 2011, 156, 213-227. [CrossRef]

9. Giri, M.K.; Swain, S.; Gautam, J.K.; Singh, S.; Singh, N.; Bhattacharjee, L.; Nandi, A.K. The Arabidopsis thaliana At4g13040 gene, a unique member of the $A P 2 /$ EREBP family, is a positive regulator for salicylic acid accumulation and basal defense against bacterial pathogens. J. Plant Physiol. 2014, 171, 860-867. [CrossRef]

10. Sakuma, Y.; Liu, Q.; Dubouzet, J.G. DNA-binding specificity of the ERF/AP2 domain of Arabidopsis DREBs, transcription factors involved in dehydrationand cold-inducible gene expression. Biochem. Biophys. Res. Commun. 2002, 290, 998-1009. [CrossRef]

11. Sharoni, A.M.; Nuruzzaman, M.; Satoh, K. Gene structures, classification and expression models of the AP2/ERF transcription factor family in rice. Plant Cell Physiol. 2011, 52, 344-360. [CrossRef] [PubMed]

12. Zhuang, J.; Cai, B.; Peng, R.H. Genome-wide analysis of the AP2/ERF gene family in Populus trichocarpa. Biochem. Biophys. Res. Commun. 2008, 371, 468-474. [CrossRef] [PubMed]

13. Licausi, F.; Giorgi, F.M.; Zenoni, S. Genomic and transcriptomic analysis of the AP2/ERF superfamily in Vitis vinifera. BMC Genom. 2010, 11, 719. [CrossRef] [PubMed]

14. Hu, L.; Liu, S. Genome-wide identification and phylogenetic analysis of the ERF gene family in cucumbers. Genet. Mol. Biol. 2011, 34, 624-633. [CrossRef]

15. Li, P.; Chai, Z.; Lin, P. Genome-wide identification and expression analysis of $A P 2 / E R F$ transcription factors in sugarcane (Saccharum spontaneum L.). BMC Genom. 2020, 21, 685. [CrossRef] [PubMed]

16. Zhang, Z.; Li, X. Genome-wide identification of $A P 2 / E R F$ supERFamily genes and their expression during fruit ripening of Chinese jujube. Sci. Rep. 2018, 8, 15612. [CrossRef]

17. Zhang, G.; Chen, M.; Chen, X. Phylogeny, gene structures, and expression patterns of the ERF gene family in soybean (Glycine max L.). J. Exp. Bot. 2008, 59, 4095-4107. [CrossRef] [PubMed]

18. Sharma, M.K.; Kumar, R.; Solanke, A.U. Identification, phylogeny, and transcript profiling of ERF family genes during development and abiotic stress treatments in tomato. Mol. Genet. Genom. 2010, 284, 455-475. [CrossRef] [PubMed]

19. Zhuang, J.; Chen, J.M.; Yao, Q.H. Discovery and expression profile analysis of AP2/ERF family genes from Triticum aestivum. Mol. Biol. Rep. 2011, 38, 745-753. [CrossRef]

20. Li, H.; Wang, Y.; Wu, M.; Li, L.; Li, C.; Han, Z.; Yuan, J.; Chen, C.; Song, W.; Wang, C. Genome-wide identification of AP2 / ERF transcription factors in cauliflower and expression profiling of the ERF family under salt and drought stresses. Front. Plant Sci. 2017, 8, 946. [CrossRef] 
21. Yamada, Y.; Nishida, S.; Shitan, N. Genome-wide identification of AP2/ERF transcription factor-encoding genes in California poppy (Eschscholzia californica) and their expression profiles in response to methyl jasmonate. Sci. Rep. 2020, 10, 18066. [CrossRef] [PubMed]

22. Xu, L.; Feng, G.; Yang, Z.; Xu, X.; Huang, L.; Yang, Q.; Zhang, X. Genome-wide AP2/ERF gene family analysis reveals the classification, structure, expression profiles and potential function in orchardgrass (Dactylis glomerata). Mol. Biol. Rep. 2020, 47, 5225-5241. [CrossRef] [PubMed]

23. Cao, S.; Wang, Y.; Li, X.; Gao, F.; Feng, J.; Zhou, Y. Characterization of the AP2/ERF Transcription Factor Family and Expression Profiling of DREB Subfamily under Cold and Osmotic Stresses in Ammopiptanthus nanus. Plants 2020, 9, 455. [CrossRef]

24. Najafi, S.; Sorkheh, K.; Nasernakhaei, F. Characterization of the APETALA2/Ethylene-responsive factor (AP2/ERF) transcription factor family in sunflower. Sci. Rep. 2018, 8, 11576. [CrossRef] [PubMed]

25. Dietz, K.J.; Vogel, M.O.; Viehhauser, A. AP2/EREBP transcription factors are part of gene regulatory networks and integrate metabolic, hormonal and environmental signals in stress acclimation and retrograde signalling. Protoplasma 2010, $245,3-14$. [CrossRef] [PubMed]

26. Zhou, M.Q.; Shen, C.; Wu, L.H. CBF-dependent signaling pathway: A key responder to low temperature stress in plants. Crit. Rev. Biotechnol. 2011, 31, 186-192. [CrossRef]

27. Yu, Y.; Liu, A.; Duan, X. GsERF6, an ethyleneresponsive factor from Glycine soja, mediates the regulation of plant bicarbonate tolerance in Arabidopsis. Planta 2016, 244, 681-698. [CrossRef]

28. Xie, Z.; Nolan, T.; Jiang, H. The AP2/ERF Transcription factor TINY modulates brassinosteroid regulated plant growth and drought responses in Arabidopsis. Plant Cell 2019, 31, 1788-1806. [CrossRef]

29. Feng, W.; Li, J.; Long, S. A DREB1 gene from zoysiagrass enhances Arabidopsis tolerance to temperature stresses without growth inhibition. Plant Sci. 2019, 278, 20-31. [CrossRef]

30. Javed, T.; Shabbir, R.; Ali, A.; Afzal, I.; Zaheer, U.; Gao, S.J. Transcription Factors in Plant Stress Responses: Challenges and Potential for Sugarcane Improvement. Plants 2020, 9, 491. [CrossRef]

31. Wang, S.; Guo, T.; Wang, Z.; Kang, J.; Yang, Q.; Shen, Y.; Long, R. Expression of three related to ABI3/VP1 genes in Medicago truncatula caused increased stress resistance and branch increase in Arabidopsis thaliana. Front. Plant Sci. 2020, 11, 611. [CrossRef]

32. Kavas, M.; Gökdemir, G.; Seçgin, Z.; Bakhsh, A. Ectopic expression of common bean ERF transcription factor PvERF35 promotes salt stress tolerance in tobacco. Plant Biol. 2020, 22, 1102-1112. [CrossRef]

33. Lv, K.; Li, J.; Zhao, K.; Chen, S.; Nie, J.; Zhang, W.; Liu, G.; Wei, H. Overexpression of an AP2/ERF family gene, BpERF13, in birch enhances cold tolerance through upregulating CBF genes and mitigating reactive oxygen species. Plant Sci. 2020, 292, 110375 [CrossRef] [PubMed]

34. Sun, X.; Zhu, Z.; Zhang, L.; Fang, L.; Zhang, J.; Wang, Q.; Li, S.; Liang, Z.; Xin, H. Overexpression of ethylene response factors VaERF080 and VaERF087 from Vitis amurensis enhances cold tolerance in Arabidopsis. Sci. Hortic. 2019, 243, 320-326. [CrossRef]

35. Moon, S.J.; Min, M.K.; Kim, J.; Kim, D.Y.; Yoon, I.S.; Kwon, T.R.; Byun, M.O.; Kim, B.G. Ectopic expression of OsDREB1G, a member of the OsDREB1 subfamily, confers cold stress tolerance in rice. Front. Plant Sci. 2019, 10, 297. [CrossRef] [PubMed]

36. Jin, Y.; Pan, W.; Zheng, X. OsERF101, an ERF family transcription factor, regulates drought stress response in reproductive tissues. Plant Mol. Biol. 2018, 98, 51-65. [CrossRef] [PubMed]

37. Hwang, J.E.; Lim, C.J.; Chen, H. Overexpression of Arabidopsis dehydration- responsive element-binding protein 2C confers tolerance to oxidative stress. Mol. Cells 2012, 33, 135-140. [CrossRef] [PubMed]

38. Wang, C.T.; Dong, Y.M. Overexpression of maize ZmDBP3 enhances tolerance to drought and cold stress in transgenic Arabidopsis plants. Biologia 2009, 64, 1108. [CrossRef]

39. Zhu, J.K. Salt and drought stress signal transduction in plants. Annu. Rev. Plant Biol. 2002, 53, 247-273. [CrossRef] [PubMed]

40. EPOA. Palm Oil Production; EPOA: Brussels, Belgium, 2019.

41. Barcelos, E.; Rios, S.A.; Cunha, R.N. Oil palm natural diversity and the potential for yield improvement. Front. Plant Sci. 2015, 6, 190. [CrossRef] [PubMed]

42. Zhou, L.; Yarra, R.; Jin, L.; Cao, H.X. Genome-wide identification and expression analysis of MYB gene family in oil palm (Elaeis guineensis Jacq.) under abiotic stress conditions. Environ. Exp. Bot. 2020, 180, 104245. [CrossRef]

43. Murugesan, P.; Aswathy, G.M.; Kumar, S.K.; Masilamani, P.; Kumar, V.; Ravi, V. Oil palm (Elaeis guineensis) genetic resources for abiotic stress tolerance: A review. Ind. J. Agric. Sci. 2017, 87, 571-579.

44. Xiao, Y.; Zhou, L.; Lei, X.; Cao, H.; Wang, Y.; Dou, Y. Genome-wide identification of WRKY genes and their expression profiles under different abiotic stresses in Elaeis guineensis. PLoS ONE 2017, 12, e0189224. [CrossRef]

45. Abdullah, S.N.A.; Azzeme, A.M.; Ebrahimi, M.; Ariff, E.A.K.E.; Hanifiah, F.H.A. Transcription Factors Associated with Abiotic Stress and Fruit Development in Oil Palm. In Crop Improvement; Abdullah, S., Chai-Ling, H., Wagstaff, C., Eds.; Springer: Cham, Switzerland, 2017.

46. Qaim, M.; Sibhatu, K.T.; Siregar, H.; Grass, I. Environmental, economic, and social consequences of the oil palm boom. Ann. Rev. Res. Econ. 2020, 12, 321-344. [CrossRef]

47. Cui, L.; Feng, K.; Wang, M.; Wang, M.; Deng, P.; Song, W.; Nie, X. Genome-wide identification, phylogeny and expression analysis of AP2 / ERF transcription factors family in Brachypodium distachyon. BMC Genom. 2016, 17, 636. [CrossRef]

48. Wu, H.; Lv, H.; Li, L.; Liu, J.; Mu, S.; Li, X. Genome-wide analysis of the AP2/ERF transcription factors family and the expression patterns of DREB genes in Moso bamboo (phyllostachys edulis). PLoS ONE 2015, 10, e0126657. [CrossRef] [PubMed] 
49. Xu, W.; Li, F.; Ling, L.; Liu, A. Genome-wide survey and expression profiles of the AP2/ERF family in castor bean (Ricinus communis L.). BMC Genom. 2013, 14, 785. [CrossRef]

50. Boeva, V. Analysis of genomic sequence motifs for deciphering transcription factor binding and transcriptional regulation in eukaryotic cells. Front. Genet. 2016, 7, 24. [CrossRef]

51. Faraji, S.; Filiz, E.; Kazemitabar, S.K.; Vannozzi, A.; Palumbo, F.; Barcaccia, G.; Heidari, P. The AP2/ERF Gene Family in Triticum durum: Genome-wide identification and expression analysis under drought and salinity stresses. Genes 2020, 11, 1464. [CrossRef]

52. Shu, Y.; Liu, Y.; Zhang, J.; Song, L.; Guo, C. Genome-wide analysis of the AP2/ERF superfamily genes and their responses to abiotic stress in Medicago truncatula. Front. Plant Sci. 2016, 6, 1247. [CrossRef] [PubMed]

53. Huang, Y.; Liu, Y.; Zhang, M.; Chai, M.; He, Q.; Jakada, B.H.; Chen, F.; Chen, H.; Jin, X.; Cai, H. Genome-wide identification and expression analysis of the ERF transcription factor family in pineapple (Ananas comosus (L.) Merr.). Peer J. 2020, 8, e10014. [CrossRef] [PubMed]

54. Sohn, K.H.; Lee, S.C.; Jung, H.W.; Hong, J.K.; Hwang, B.K. Expression and functional roles of the pepper pathogen-induced transcription factor $R A V 1$ in bacterial disease resistance, and drought and salt stress tolerance. Plant Mol. Biol. 2006, 61, 897-915. [CrossRef] [PubMed]

55. Abiri, R.; Shaharuddin, N.A.; Maziah, M.; Yusof, Z.N.B.; Atabaki, N.; Sahebi, M. Role of ethylene and the APETALA 2/ethylene response factor superfamily in rice under various abiotic and biotic stress conditions. Environ. Exp. Bot. 2017, 134, 33-44. [CrossRef]

56. Wang, Y.; Tang, H.; DeBarry, J.D.; Tan, X.; Li, J.; Wang, X.; Lee, T.; Jin, H.; Marler, B.; Guo, H.; et al. MCScanX: A toolkit for detection and evolutionary analysis of gene synteny and collinearity. Nucl. Acids Res. 2012, 40, e49. [CrossRef] 